\title{
Can laboratory assays for measurement of cellular DNA damage and its repair be useful for prediction of cancer and prognosis?
}

\author{
William $\mathrm{Au}^{1,2^{*}}$ \\ 1. Shantou University Medical College, Shantou, China \\ 2. CCAMF, University of Medicine and Pharmacy, Tirgu Mures, Romania
}

\section{Introduction}

For the development of cancer, it is generally accepted that certain key events are frequently involved. Two of them are the expression of DNA damage and inadequate repair of the damage. Expression of different types of DNA damage, particularly after exposure to environmental mutagens and carcinogens, have been well-characterized. Examples of the different types of DNA damages are shown in Figure 1 and each type of damage requires repair from specific and genetically-controlled DNA repair pathways. DNA single- and double-strand breaks from exposure to X-rays, for example, require the non-homologous end-joining and/or homologous recombination repair pathways. Repair of pyrimidine dimers from exposure to UV-light requires the excision repair pathway. Among the different types of repair activities, the repair of DNA double-strand breaks is most troublesome to cells because there is not a homologous DNA strand to provide complementary sequences for the repair therefore mistakes are frequently made as a consequence of the repair of DNA double-strand breaks.

The often observed consequence from induction of DNA damage and inadequate repair of the damage is expression of chromosome abnormalities, gene mutations and genomic instability in most cancer cells. Therefore, better characterization of DNA damage and its repair activities can provide more precise determination of cancer and of prognosis.

\section{Assays for DNA damage}

The usefulness of the standard cytogenetic assay to determine quality and quantity of chromosome abnormalities have been well-characterized and validated (1). As mentioned earlier, most cancer cells show the presence of chromo-

*Corresponding author: CCAMF, University of Medicine and Pharmacy Tirgu Mures, 38 Gh Marinescu str, Romania, e-mail: william.au@umftgm.ro 


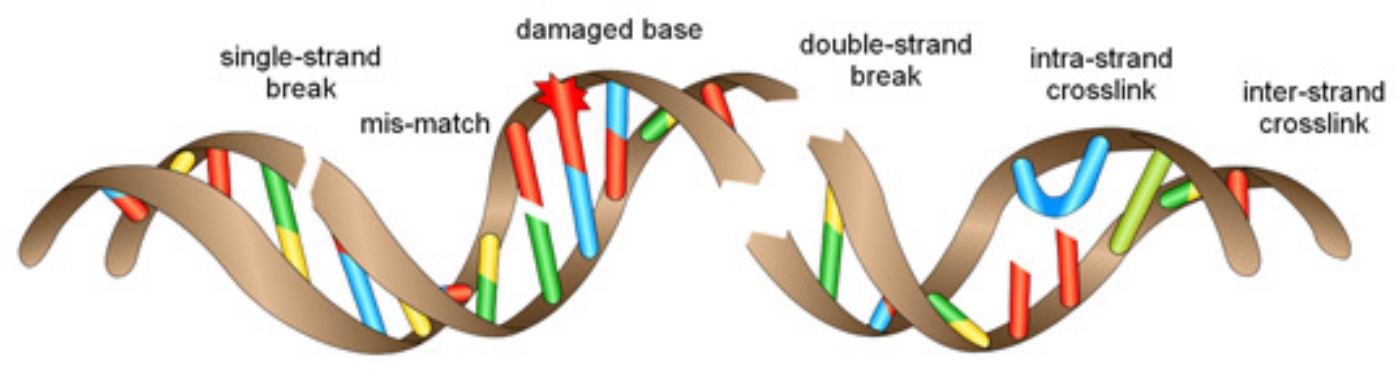

Fig. 1. Different types of DNA damage (acquired from "sites.google.com"; accessed November 10, 2017).

some abnormalities. In addition, some abnormalities, such as translocation between chromosomes 8 and 22, and deletion in chromosome 13 have been used as diagnostic markers for their respective cancers, i.e. chronic myelogenous leukemia and retinoblastoma. Furthermore, many disease-specific chromosome abnormalities are responsible for unique and abnormal gene expressions. Consequently, such information becomes critically useful for the development of mechanism- and molecular-based therapeutic decisions, as well as for prognosis follow-up.

Besides using the standard cytogenetic assay to determine abnormalities in cancer cells, the assay has also been used frequently to determine excessive exposure to mutagens and carcinogens. The generally accepted scenario is that observation of exposure-induced chromosome abnormalities in normal populations is indicative of the population's increased risk for development of cancer since most cancer cells also contain chromosome abnormalities. However, the reality is not this straight forward because many more key events must occur during the multi-stage and prolonged developmental process before cancer cells can be formed. Consequently, even among an exposed population which has significant increase of exposure-induced chromosome abnormalities, only very few individuals would develop cancer eventually. Nevertheless, the assay is valuable in providing evidence to help with the development of disease prevention and of environmental health policy decisions.

In addition to the use of cytogenetic assay to monitor exposed populations, other assays have also been developed and used. Examples are the micronuclei, Comet and $\mathrm{rH} 2 \mathrm{AX}$ assays. The micronuclei assay, as illustrated in the name, is used to determine the frequency of intracellular small nuclei besides the main nuclei (2). Therefore, the assay typically determines the induction and presence of chromosome breaks. The Comet assay has been used to determine the frequency of single- and/or double-strand DNA breaks or DNA damage which can lead to the expression of DNA breaks (3). The $\mathrm{rH} 2 \mathrm{AX}$ assay determine presence of DNA strand breaks because it is based on measurement of the amount of phosphorylation of histone $2 \mathrm{AX}$ which binds to and accumulate at sites of DNA strand breaks (4). 
Although these three newer assays are less laborious to conduct than the cytogenetic assay, their application towards health risk assessment needs to be better characterized. Several important questions need to be addressed. For example, what is the consequence of cells that have micronuclei? Since micronuclei contain mostly broken pieces of chromosomes, these cells would probably not survive. However, one may assume that cells with small amount of chromosome damage may survive. As shown in the cytogenetic assay, chromosome translocations and interstitial deletions would allow cells to survive and these abnormalities are frequently observed in cancer cells. The specific chromosome abnormalities are probably caused by mistakes in repair of the initial DNA damage.

DNA strand breaks which are readily detected by the Comet and the $\mathrm{rH} 2 \mathrm{AX}$ assays are quickly repaired within cells. However, mistakes in the repair of strand breaks, especially double-strand breaks, are the crucial events which can cause serious consequences.

\section{Measurement of DNA repair capacity in human populations and assessment of health risk}

As described earlier, mistakes in repair of DNA damage is a key event which can cause serious health consequences such as the development of cancer (5). In the human population, individuals who have inherited abnormal genes which cause serious deficiency in DNA repair can develop major health problems. Examples of these are patients who have Chromosome Insta- bility and Xeroderma pigmentosum sydromes. The latter patients have been well-characterized to have deficiency in the repair of UV-light induced pyrimidine dimers and to have high frequencies for development of skin cancers.

Besides patients with inherent DNA repair defects, detecting reduced DNA repair capacity (e.g. from gene polymorphisms) in normal individuals is very difficult. This is because any repair deficiency would be minimal among normal people and most existing DNA repair assays are insensitive for detecting such small deficiency. Therefore, functions of most polymorphisms in DNA repair genes have not been characterized in normal people yet.

The Challenge assay which was developed by the author is capable of detecting small differences in DNA repair capacity among normal individuals (1, 5). Briefly, lymphocytes from donors are cultured and irradiated with X-rays or UV-light to induce DNA damage in vitro. Therefore, these cells are challenged to repair the induced damage. At appropriate time, the irradiated cells are harvested to determine the frequency of chromosome abnormalities, e.g. translations using the cytogenetic assay, or the lack of reduction of DNA strand breaks using the Comet assay.

Several studies show that the assay is both sensitive and specific for determining different types of DNA repair capacity among normal populations, i.e. base-excision and nucleotide-excision repair for X-ray and UV-light induced DNA damage $(1,5)$. Consequently, the assay is used around the world for determining DNA repair 
deficiency in normal and exposed human populations and for assessment of health risk.

The Challenge-comet assay is also used in patients. In a study of breast cancer patients in China, patients were found to have quantitatively and significantly lower DNA repair capacity than matched normal females (6). Therefore, the data suggest that, among normal females, those who have lower DNA repair capacity are at higher risk for the development of breast cancer. Of course, the increased risk is also contributed by other risk factors. The study also had a follow-up on the breast cancer patients. Among the patients, those with lower DNA repair capacity were found to have worse prognosis, i.e. metastasis.

\section{Conclusion}

Laboratory techniques have been relied upon to provide data for diagnosis of disease and for determination of therapeutic protocols. This editorial provides an emphasis on significant value which can be gained from further improvement of techniques. In particular, characterization of functional DNA repair deficiency is critically useful in understanding health risk. Quantitative and functional characterization of the deficiency can potentially be used to provide personalized risk assessment, to enhance decision for thera- peutic protocols and to make prognosis evaluation.

\section{Acknowledgement}

This work was supported by the University of Medicine and Pharmacy of Tîrgu Mureș, Romania, Research Grant number 15609/13/29.12.2017"

\section{References}

1. Au WW, Salama SA, Sierra-Torres CH. Functional characterization of polymorphism in DNA repair genes using cytogenetic challenge assays. Environ Health Persp. 2003;111;1843-50. DOI: 10.1289/ehp.6632

2. Luzhna L, Kathiria P, Kovalchuk O. Micronuclei in genotoxicity assessment: from genetics to epigenetics and beyond. Front genet. 2013;4:131. DOI: 10.3389/ fgene.2013.00131

3. Au WW, Salama SA. Cytogenetic challenge assays for assessment of DNA repair capacities. In: Methods in Molecular Biology: DNA Repair Protocols: Mammalian Systems. 2nd Edition. Edited by: DS Henderson, Humana Press, Totowa, NJ., 2005; Chapter 3, 25-42.

4. Nikolova T, Dvorak M, Jung F, Adam I, Krämer E, Gerhold-Ay A, Kaina B. The $\gamma \mathrm{H} 2 \mathrm{AX}$ Assay for Genotoxic and Nongenotoxic Agents: Comparison of H2AX Phosphorylation with Cell Death Response, Toxicol Sci. 2014;140(1):103-17. DOI: 10.1093/toxsci/kfu066

5. Rukirawat M, Cebulska-Wasilewska A, Au WW. Evidence for exposure-induced DNA repair abnormality is indicative of health and genetic risk, Int J Hygiene Env Health. 2013;216:566-73. DOI: 10.1016/j. ijheh.2013.03.003

6. Fu XJ, Shi XJ, Lin K, Lin R, Huang WH, Zhang GJ, $\mathrm{Au}$ WW. Environmental and DNA repair risk factors for breast cancer in Southern China. Int J Hygiene Env Health. 2015;218:313-318. DOI: 10.1016/j. ijheh.2015.01.001 